\title{
Initial logarithmic coefficients for functions starlike with respect to symmetric points
}

\section{Paweł Zaprawa ${ }^{1}$ (D)}

Received: 26 April 2021 / Accepted: 16 July 2021 / Published online: 2 August 2021

(C) The Author(s) 2021

\begin{abstract}
In this paper, we obtain the bounds of the initial logarithmic coefficients for functions in the classes $\mathcal{S}_{S}^{*}$ and $\mathcal{K}_{S}$ of functions which are starlike with respect to symmetric points and convex with respect to symmetric points, respectively. In our research, we use a different approach than the usual one in which the coeffcients of $f$ are expressed by the corresponding coeffcients of functions with positive real part. In what follows, we express the coeffcients of $f$ in $\mathcal{S}_{S}^{*}$ and $\mathcal{K}_{S}$ by the corresponding coeffcients of Schwarz functions. In the proofs, we apply some inequalities for these functions obtained by Prokhorov and Szynal, by Carlson and by Efraimidis. This approach offers a additional benefit. In many cases, it is easily possible to predict the exact result and to select extremal functions. It is the case for $\mathcal{S}_{S}^{*}$ and $\mathcal{K}_{S}$.
\end{abstract}

Keywords Univalent functions · Logarithmic coefficients

Mathematics Subject Classification $30 \mathrm{C} 45 \cdot 30 \mathrm{C} 50$

\section{Introduction}

Let $\mathbb{D}$ be the unit disk $\{z \in \mathbb{C}:|z|<1\}$ and $\mathcal{A}$ be the family of all functions $f$ analytic in $\mathbb{D}$, normalized by the condition $f(0)=f^{\prime}(0)-1=0$. It means that $f$ has the expansion

$$
f(z)=z+\sum_{n=2}^{\infty} a_{n} z^{n} .
$$

Let $\mathcal{S}$ be the class of all functions in $\mathcal{A}$ which are univalent and $\mathcal{S}^{*}$ be the subset of $\mathcal{S}$

Paweł Zaprawa

p.zaprawa@pollub.pl

1 Department of Mathematics, Faculty of Mechanical Engineering, Lublin University of Technology, Nadbystrzycka 36, 20-618 Lublin, Poland 
consisting of those functions which are starlike in $\mathbb{D}$. The logarithmic coefficients of $f \in \mathcal{S}$, denoted by $\gamma_{n}=\gamma_{n}(f)$, are defined by

$$
\frac{1}{2} \log \frac{f(z)}{z}=\sum_{n=1}^{\infty} \gamma_{n} z^{n}
$$

If $f$ is given by (1.1), then its logarithmic coefficients are given by

$$
\begin{aligned}
& \gamma_{1}=\frac{1}{2} a_{2} \\
& \gamma_{2}=\frac{1}{2}\left(a_{3}-\frac{1}{2} a_{2}^{2}\right) \\
& \gamma_{3}=\frac{1}{2}\left(a_{4}-a_{2} a_{3}+\frac{1}{3} a_{2}^{3}\right) \\
& \gamma_{4}=\frac{1}{2}\left(a_{5}-a_{2} a_{4}+a_{2}^{2} a_{3}-\frac{1}{2} a_{3}^{2}-\frac{1}{4} a_{2}^{4}\right) \\
& \gamma_{5}=\frac{1}{2}\left(a_{6}-a_{2} a_{5}-a_{3} a_{4}+a_{2} a_{3}^{2}+a_{2}^{2} a_{4}-a_{2}^{3} a_{3}+\frac{1}{5} a_{2}^{5}\right) .
\end{aligned}
$$

It is known that for the Koebe function $f(z)=\frac{z}{(1-z)^{2}}$ there is $\gamma_{n}=\frac{1}{n}$ for each positive integer $n$. Since the Koebe function appears as an extremal function in many problems of geometric theory of analytic functions, one could expect that $\left|\gamma_{n}\right| \leq \frac{1}{n}$ for each $f \in \mathcal{S}$ and $n \in \mathbb{N}$. It is not true even for $\gamma_{2}$. It is enough to use the Fekete-Szegö inequality

$$
\left|a_{3}-\mu a_{2}^{2}\right| \leq 1+2 e^{-\frac{2 \mu}{1-\mu}}
$$

which holds for all $f \in \mathcal{S}$ and $0 \leq \mu<1$. Consequently, the following sharp bound $\left|\gamma_{2}\right| \leq \frac{1}{2}\left(1+2 e^{-2}\right)=0.635 \ldots$ is valid for $\mathcal{S}$. A little is known about succeeding logarithmic coefficients of univalent functions. In very recent paper [5], Obradović and Tuneski proved that $\left|\gamma_{3}\right| \leq \frac{\sqrt{133}}{15}$ for all $f \in \mathcal{S}$.

Girela in [3] shown that also in the class $\mathcal{C}$ of close-to-convex functions there exist functions such that their logarithmic coefficients are greater than $\frac{1}{n}$. The similar fact, but for the class $\mathcal{U}$ of univalent functions satisfying the condition

$$
\left|\left(\frac{z}{f(z)}\right)^{2} f^{\prime}(z)-1\right|<1
$$

was proved by Obradović et al. in [4]. On the other hand, the inequality $\left|\gamma_{n}\right| \leq \frac{1}{n}$ holds for each $f \in \mathcal{S}^{*}$. For a summary of some of the significant results concerning the logarithmic coefficients for univalent functions, see [8].

In this paper, we consider two subclasses of $\mathcal{S}$ : the class $\mathcal{S}_{S}^{*}$ of functions starlike with respect to the symmetric points and the relative class $\mathcal{K}_{S}$ of functions convex with respect to the symmetric points. The definitions are as follows

$$
\mathcal{S}_{S}^{*}=\left\{f \in \mathcal{S}: \operatorname{Re}\left\{\frac{2 z f^{\prime}(z)}{f(z)-f(-z)}\right\}>0, \quad z \in \Delta\right\}
$$

and 


$$
\mathcal{K}_{S}=\left\{f \in \mathcal{S}: \operatorname{Re}\left\{\frac{2\left[z f^{\prime}(z)\right]^{\prime}}{[f(z)-f(-z)]^{\prime}}\right\}>0, \quad z \in \Delta\right\}
$$

It is known (see, [7]) that $\mathcal{K} \subset \mathcal{S}_{S}^{*}$ and $\mathcal{S}^{*^{(2)}} \subset \mathcal{S}_{S}^{*}$, where $\mathcal{S}^{*^{(2)}}$ stands for the class of odd starlike functions. On the other hand, $\mathcal{S}_{S}^{*} \subset \mathcal{C}$.

Our aim is to derive the bounds of the initial logarithmic coefficients for functions in the both classes defined above. It is worth observing that the Koebe function does not belong to $\mathcal{S}_{S}^{*}$, so it cannot play a role of extremal function for $\mathcal{S}_{S}^{*}$.

In our research, we use a different approach than the usual one in which the coeffcients of $f$ are expressed by the corresponding coeffcients of functions with positive real part. In what follows, we express the coeffcients of $f$ in $\mathcal{S}_{S}^{*}$ and $\mathcal{K}_{S}$ by the corresponding coeffcients of Schwarz functions. This makes the calculation easier. Additionally, this approach offers a valuable benefit. In many cases, it is easily possible to predict the exact result and to select extremal functions. It is the case for $\mathcal{S}_{S}^{*}$ and $\mathcal{K}_{S}$.

Let $\mathcal{B}_{0}$ be the class of Schwarz functions, i.e., analytic functions $\omega: \mathbb{D} \rightarrow \mathbb{D}$, $\omega(0)=0$. A function $\omega \in \mathcal{B}_{0}$ can be written as a power series

$$
\omega(z)=\sum_{n=1}^{\infty} c_{n} z^{n}
$$

To prove our results, we need the following lemmas for Schwarz functions obtained by Prokhorov and Szynal [6], by Carlson ([1]) and by Efraimidis [2].

Lemma 1 Let $\omega(z)=c_{1} z+c_{2} z^{2}+\cdots$ be a Schwarz function. Then, for any real numbers $\mu$ and $v$ such that

$$
(\mu, v) \in\left\{\frac{1}{2} \leq|\mu| \leq 2, \frac{4}{27}(|\mu|+1)^{3}-(|\mu|+1) \leq v \leq 1\right\},
$$

the following sharp estimate holds

$$
\left|c_{3}+\mu c_{1} c_{2}+v c_{1}^{3}\right| \leq 1
$$

Lemma 2 Let $\omega(z)=c_{1} z+c_{2} z^{2}+\cdots$ be a Schwarz function. Then

$$
\left|c_{2}\right| \leq 1-\left|c_{1}\right|^{2} \quad, \quad\left|c_{3}\right| \leq 1-\left|c_{1}\right|^{2}-\frac{\left|c_{2}\right|^{2}}{1+\left|c_{1}\right|} \quad, \quad\left|c_{4}\right| \leq 1-\left|c_{1}\right|^{2}-\left|c_{2}\right|^{2} .
$$

Lemma 3 Let $\omega(z)=c_{1} z+c_{2} z^{2}+\cdots$ be a Schwarz function. Then

$$
\left|c_{4}+2 c_{1} c_{3}+c_{2}^{2}+3 c_{1}^{2} c_{2}+c_{1}^{4}\right| \leq 1 \text {. }
$$


Lemma 3 is a particular case of more general theorem which is needed in our proofs. For $p$ in $\mathcal{P}$, the class of analytic functions $p$ such that $\operatorname{Rep}(z)>0$ and $p(0)=1$, and for $w \in \mathbb{C}$, Efraimidis in [2] defined the determinant

$$
A_{k, n}(w)=\left|\begin{array}{cccccc}
p_{n+k} & p_{n+k-1} & p_{n+k-2} & \ldots & p_{n+1} & p_{n} \\
w p_{1} & 1 & 0 & \ldots & 0 & 0 \\
w p_{2} & w p_{1} & 1 & \ldots & 0 & 0 \\
\ddots & \ddots & \ddots & \vdots & \ddots & \ddots \\
w p_{k-1} & w p_{k-2} & w p_{k-3} & \ldots & 1 & 0 \\
w p_{k} & w p_{k-1} & w p_{k-2} & \ldots & w p_{1} & 1
\end{array}\right|
$$

and proved the following theorem.

Theorem 1 If $p \in \mathcal{P}$ and $w \in \mathbb{C}$, then

$$
\left|A_{k, n}(w)\right| \leq 2 \max \left\{1,|1-2 w|^{k}\right\}
$$

for all integers $k \geq 0$ and $n \geq 1$.

Applying the correspondence between $p \in \mathcal{P}$ and $\omega \in \mathcal{B}_{0}$,

$$
p(z)=\frac{1+\omega(z)}{1-\omega(z)}
$$

it is possible to obtain the analogous theorem for Schwarz functions. As a corollary, putting $w=0$ and $k=3, n=1$, Lemma 3 follows.

Consider now the case $k+n=5$ in Theorem 1. Formula (1.5) and (1.6) result in Corollary 1 If $\omega \in \mathcal{B}_{0}$ is of the form (1.4) and $\mu \in \mathbb{C},|\mu| \leq 1$, then

$$
\begin{gathered}
\left|c_{5}+2 \mu c_{1} c_{4}+2 \mu c_{2} c_{3}+3 \mu^{2} c_{1} c_{2}^{2}+3 \mu^{2} c_{1}^{2} c_{3}+4 \mu^{3} c_{1}^{3} c_{2}+\mu^{4} c_{1}^{5}\right| \leq 1 \\
\mid c_{5}+(1+\mu) c_{1} c_{4}+2 \mu c_{2} c_{3}+\mu(1+2 \mu) c_{1} c_{2}^{2}+\mu(2+\mu) c_{1}^{2} c_{3} \\
+\mu^{2}(3+\mu) c_{1}^{3} c_{2}+\mu^{3} c_{1}^{5} \mid \leq 1 \\
\mid c_{5}+(1+\mu) c_{1} c_{4}+(1+\mu) c_{2} c_{3}+3 \mu c_{1} c_{2}^{2}+\left(1+\mu+\mu^{2}\right) c_{1}^{2} c_{3} \\
+2 \mu(1+\mu) c_{1}^{3} c_{2}+\mu^{2} c_{1}^{5} \mid \leq 1 .
\end{gathered}
$$

Combining Formulae (1.7-1.9) for suitably chosen $\mu$ we can get other relative inequalities; some of them will be useful in proving theorems from the two next sections.

Taking $\mu=0$ and $\mu=-1$ in (1.9), we have

$$
\left|c_{5}+c_{1} c_{4}+c_{2} c_{3}+c_{1}^{2} c_{3}\right| \leq 1
$$

and 


$$
\left|c_{5}-3 c_{1} c_{2}^{2}+c_{1}^{2} c_{3}+c_{1}^{5}\right| \leq 1 .
$$

From (1.7) with $\mu=1$ and $\mu=-1$, it follows that

$$
\left|c_{5}+3 c_{1} c_{2}^{2}+3 c_{1}^{2} c_{3}+c_{1}^{5}\right| \leq 1 .
$$

Finally, (1.11) and (1.12) results in

$$
\left|c_{5}+2 c_{1}^{2} c_{3}+c_{1}^{5}\right| \leq 1 .
$$

\section{Logarithmic coefficients for functions in $\mathcal{S}_{S}^{*}$}

Although the first three results in the theorem below are easy to obtain in other way, for completeness of results, we prove all of them in a uniform way.

Theorem 2 If $f \in \mathcal{S}_{S}^{*}$, then

$$
\left|\gamma_{1}\right| \leq \frac{1}{2} \quad, \quad\left|\gamma_{2}\right| \leq \frac{1}{2} \quad, \quad\left|\gamma_{3}\right| \leq \frac{1}{4} \quad, \quad\left|\gamma_{4}\right| \leq \frac{1}{4} \quad, \quad\left|\gamma_{5}\right| \leq \frac{1}{6}
$$

All bounds are sharp.

Proof If $f \in \mathcal{S}_{S}^{*}$, then

$$
\frac{2 z f^{\prime}(z)}{f(z)-f(-z)}=\frac{1+\omega(z)}{1-\omega(z)},
$$

where $\omega \in \mathcal{B}_{0}$. Using (1.1) and (1.4) and comparing coefficients at powers of $z$ in

$$
2 z f^{\prime}(z)(1-\omega(z))=\frac{1}{2}[f(z)-f(-z)](1+\omega(z))
$$

we get

$$
\begin{aligned}
& a_{2}=c_{1} \\
& a_{3}=c_{2}+c_{1}^{2} \\
& a_{4}=\frac{1}{2}\left(c_{3}+3 c_{1} c_{2}+2 c_{1}^{3}\right) \\
& a_{5}=\frac{1}{2}\left(c_{4}+2 c_{1} c_{3}+2 c_{2}^{2}+5 c_{1}^{2} c_{2}+2 c_{1}^{4}\right) \\
& a_{6}=\frac{1}{3}\left(c_{5}+\frac{5}{2} c_{1} c_{4}+3 c_{2} c_{3}+6 c_{1} c_{2}^{2}+5 c_{1}^{2} c_{3}+\frac{19}{2} c_{1}^{3} c_{2}+3 c_{1}^{5}\right) .
\end{aligned}
$$

Applying (2.1) in (1.3), we obtain 


$$
\begin{aligned}
& \gamma_{1}=\frac{1}{2} c_{1} \\
& \gamma_{2}=\frac{1}{2}\left(c_{2}+\frac{1}{2} c_{1}^{2}\right) \\
& \gamma_{3}=\frac{1}{4}\left(c_{3}+c_{1} c_{2}+\frac{2}{3} c_{1}^{3}\right) \\
& \gamma_{4}=\frac{1}{4}\left(c_{4}+c_{1} c_{3}+c_{2}^{2}+2 c_{1}^{2} c_{2}+\frac{1}{2} c_{1}^{4}\right) \\
& \gamma_{5}=\frac{1}{6}\left(c_{5}+c_{1} c_{4}+\frac{3}{2} c_{2} c_{3}+\frac{3}{2} c_{1} c_{2}^{2}+2 c_{1}^{2} c_{3}+2 c_{1}^{3} c_{2}+\frac{3}{5} c_{1}^{5}\right)
\end{aligned}
$$

Since $\left|c_{1}\right| \leq 1$ and $\left|c_{2}\right| \leq 1-\left|c_{1}\right|^{2}$, the bounds of $\gamma_{1}$ and $\gamma_{2}$ are obvious. Taking $\mu=1$ and $v=2 / 3$ in Lemma 1 , the bound of $\gamma_{3}$ follows.

To derive the bound of $\gamma_{4}$, we can write

$$
\gamma_{4}=\frac{1}{8}\left[\left(c_{4}+2 c_{1} c_{3}+c_{2}^{2}+3 c_{1}^{2} c_{2}+c_{1}^{4}\right)+\left(c_{4}+c_{2}^{2}+c_{1}^{2} c_{2}\right)\right] .
$$

By Lemma 3, the first expression in square brackets is bounded by 1 . Now, it is enough to apply Lemma 2 together with the triangle inequality to obtain

$$
\left|c_{4}+c_{2}^{2}+c_{1}^{2} c_{2}\right| \leq\left(1-\left|c_{1}\right|^{2}-\left|c_{2}\right|^{2}\right)+\left|c_{2}\right|^{2}+\left|c_{1}\right|^{2}\left(1-\left|c_{1}\right|^{2}\right)=1-\left|c_{1}\right|^{4} \leq 1 .
$$

In this way, $\left|\gamma_{4}\right| \leq \frac{1}{4}$.

The coefficient $\gamma_{5}$ can be rewritten as follows

$$
\begin{aligned}
\gamma_{5}= & \frac{1}{12}\left[\left(c_{5}+2 c_{1} c_{4}+2 c_{2} c_{3}+3 c_{1} c_{2}^{2}+3 c_{1}^{2} c_{3}+4 c_{1}^{3} c_{2}+c_{1}^{5}\right)\right. \\
& \left.+\left(c_{5}+c_{2} c_{3}+c_{1}^{2} c_{3}+\frac{1}{5} c_{1}^{5}\right)\right] .
\end{aligned}
$$

The first component in the square brackets is bounded by 1 . It is a simple consequence of Formula (1.7) with $\mu=1$.

The second component is equal to $W_{1}+W_{2}$, where

$$
W_{1}=\frac{1}{5}\left(c_{5}+2 c_{1}^{2} c_{3}+c_{1}^{5}\right) \quad \text { and } \quad W_{2}=\frac{4}{5}\left(c_{5}+\frac{3}{4} c_{1}^{2} c_{3}+\frac{5}{4} c_{2} c_{3}\right) .
$$

From (1.13),

$$
\left|W_{1}\right| \leq \frac{1}{5}
$$

We shall estimate $W_{2}$ using Lemma 2. Namely,

$$
\left|W_{2}\right|=\frac{4}{5}\left|c_{5}+\frac{3}{4} c_{1}^{2} c_{3}+\frac{5}{4} c_{2} c_{3}\right| \leq \frac{4}{5} H(x, y, z),
$$

where

$$
H(x, y, z)=1-x^{2}-y^{2}-\frac{z^{2}}{1+x}+\frac{3}{4} x^{2} z+\frac{5}{4} y z
$$

and $x=\left|c_{1}\right|, y=\left|c_{2}\right|, z=\left|c_{3}\right|$. Clearly, all three variables $x, y$ and $z$ are in $[0,1]$. 
The function $H$ as a quadratic function of a variable $y$ achieves its greatest value when $y=\frac{5}{8} z$. Consequently,

$$
H(x, y, z) \leq H\left(x, \frac{5}{8} z, z\right)=1-x^{2}+\frac{25}{64} z^{2}-\frac{z^{2}}{1+x}+\frac{3}{4} x^{2} z=1-g(x, z),
$$

with

$$
g(x, z)=\left(\frac{1}{1+x}-\frac{25}{64}\right) z^{2}+x^{2}\left(1-\frac{3}{4} z\right) .
$$

Since $g(x, z) \geq 0$ for $x \in[0,1]$ and $z \in[0,1]$, so

$$
H(x, y, z) \leq 1 \text {. }
$$

Taking into account (2.4-2.6),

$$
\left|W_{1}+W_{2}\right| \leq 1
$$

and consequently,

$$
\left|\gamma_{5}\right| \leq \frac{1}{6}
$$

For the sharpness of the results, it is enough to observe that taking $\omega(z)=z^{k}$ leads to equalities in each inequality in Theorem 2.

It is worth to write the extremal functions $f \in \mathcal{S}_{S}^{*}$ and the corresponding functions

$$
F(z)=\frac{1}{2} \log \left(\frac{f(z)}{z}\right)
$$

explicitly. This is easy for even logarithmic coefficients. One can check that

$$
f_{2 n}(z)=\frac{z}{\sqrt[n]{1-z^{2 n}}} \in \mathcal{S}_{S}^{*}
$$

Indeed, this function is generated from the odd starlike function $f(z)=\frac{z}{1-z^{2}}$ under the $n$-th root transformation. Since this transformation preserves starlikeness, we can see that $f_{2 n}$ given by $(2.8)$ is in $\mathcal{S}^{*^{(2)}}$. Hence, it is in $\mathcal{S}_{S}^{*}$. For $f_{2 n}$, we derive

$$
F_{2 n}(z)=-\frac{1}{2 n} \log \left(1-z^{2 n}\right)=\sum_{j=1}^{\infty} \frac{1}{2 j n} z^{2 j n}=\frac{1}{2 n} z^{2 n}+\frac{1}{4 n} z^{4 n}+\frac{1}{6 n} z^{6 n}+\ldots
$$

For $n=1$, the extremal function is $f_{1}(z)=\frac{z}{1-z}$; for this function

$$
F_{1}(z)=-\frac{1}{2} \log (1-z)=\frac{1}{2} z+\frac{1}{4} z^{2}+\frac{1}{6} z^{3}+\ldots
$$

Consider now other positive odd integers and define a function 


$$
f_{2 n+1}(z)=\frac{z\left(1+z^{2 n+1}\right)}{\sqrt[2 n+1]{1-z^{4 n+2}}}-\frac{n}{n+1} z^{2 n+2}{ }_{2} F_{1}\left(\frac{1}{2 n+1}, \frac{n+1}{2 n+1} ; 1+\frac{1}{2 n+1} ; z^{4 n+2}\right) .
$$

Observe that $f_{2 n+1}$ is a difference of the two components. Denote them by $g$ and $h$, respectively. Clearly,

$$
\frac{1}{2}[g(z)-g(-z)]=\frac{z}{\sqrt[2 n+1]{1-z^{4 n+2}}} \quad \text { and } \quad \frac{1}{2}[h(z)-h(-z)]=0 .
$$

Moreover,

$$
z g^{\prime}(z)=\frac{z}{\left(1-z^{2 n+1}\right) \sqrt[2 n+1]{1-z^{4 n+2}}} \cdot\left[1+z^{2 n+1}+2 n z^{2 n+1}\left(1-z^{2 n+1}\right)\right],
$$

so

$$
\frac{2 z g^{\prime}(z)}{g(z)-g(-z)}=\frac{1+z^{2 n+1}}{1-z^{2 n+1}}+2 n z^{2 n+1} .
$$

On the other hand, since

$$
\begin{aligned}
h(z) & =\frac{n}{n+1} \sum_{k=0}^{\infty} \frac{\left(\frac{1}{2 n+1}\right)_{k}\left(\frac{n+1}{2 n+1}\right)_{k} z^{(4 n+2) k+2 n+2}}{k !\left(1+\frac{1}{2 n+1}\right)_{k}} \\
& =\frac{n}{n+1} \sum_{k=0}^{\infty} \frac{\left(\frac{1}{2 n+1}\right)_{k} z^{(4 n+2) k+2 n+2}}{k ! \frac{2 n+1}{n+1}\left(k+\frac{n+1}{2 n+1}\right)} \\
& =n \sum_{k=0}^{\infty} \frac{\left(\frac{1}{2 n+1}\right)_{k} z^{(4 n+2) k+2 n+2}}{k ![(2 n+1) k+n+1]},
\end{aligned}
$$

we have

$$
h^{\prime}(z)=2 n \sum_{k=0}^{\infty} \frac{1}{k !}\left(\frac{1}{2 n+1}\right)_{k} z^{(4 n+2) k+2 n+1} .
$$

Combining (2.10-2.12) leads to 


$$
\begin{aligned}
\frac{2 z f_{2 n+1}^{\prime}(z)}{f_{2 n+1}(z)-f_{2 n+1}(-z)}=\frac{2 z g^{\prime}(z)-2 z h^{\prime}(z)}{g(z)-g(-z)} \\
=\frac{1+z^{2 n+1}}{1-z^{2 n+1}}+2 n z^{2 n+1}-2 n \frac{\sum_{k=0}^{\infty} \frac{1}{k !}\left(\frac{1}{2 n+1}\right)_{k} z^{(4 n+2) k+2 n+1}}{\left(1-z^{4 n+2}\right)^{-\frac{1}{2 n+1}}} \\
=\frac{1+z^{2 n+1}}{1-z^{2 n+1}}+\frac{2 n z^{2 n+1}}{\left(1-z^{4 n+2}\right)^{-\frac{1}{2 n+1}}}\left[\sum_{k=0}^{\infty} \frac{1}{k !}\left(1-\frac{1}{2 n+1}-k\right)_{k}\left(-z^{(4 n+2)}\right)^{k}\right. \\
\left.\quad-\sum_{k=0}^{\infty} \frac{1}{k !}\left(\frac{1}{2 n+1}\right)_{k} z^{(4 n+2) k}\right] \\
=\frac{1+z^{2 n+1}}{1-z^{2 n+1}}+2 n z^{2 n+1} \sqrt[2 n+1]{1-z^{4 n+2}} \\
\quad \times \sum_{k=0}^{\infty} \frac{1}{k !}\left[(-1)^{k}\left(1-\frac{1}{2 n+1}-k\right)_{k}-\left(\frac{1}{2 n+1}\right)_{k}\right] z^{(4 n+2) k}
\end{aligned}
$$

But

$$
(-1)^{k}\left(1-\frac{1}{2 n+1}-k\right)_{k}=\left(\frac{1}{2 n+1}\right)_{k},
$$

so it results in

$$
\frac{2 z f_{2 n+1}^{\prime}(z)}{f_{2 n+1}(z)-f_{2 n+1}(-z)}=\frac{1+z^{2 n+1}}{1-z^{2 n+1}} .
$$

This means that $f_{2 n+1}$ belongs to the class $\mathcal{S}_{S}^{*}$.

From (2.9), we conclude that $f_{2 n+1}$ is $(2 n+1)$-fold symmetric function, i.e. $f_{2 n+1}(\varepsilon z)=\varepsilon f_{2 n+1}(z)$ with $\varepsilon=\exp (2 \pi /(2 n+1))$ being a root of order $2 n+1$ of unity, and

$$
f_{2 n+1}(z)=z+\frac{1}{n+1} z^{2 n+2}+\ldots
$$

Hence, the corresponding function $F_{2 n+1}$ is also $(2 n+1)$-fold symmetric and

$$
F_{2 n+1}(z)=1+\frac{1}{2(n+1)} z^{2 n+1}+\ldots
$$

The above proves that the third logarithmic coefficient is equal to $1 / 4$ for $f_{3}$ and the fifth logarithmic coefficient is equal to $1 / 6$ for $f_{5}$. The natural conjecture is following

$$
\left|\gamma_{2 n}\right| \leq \frac{1}{2 n} \quad \text { and } \quad\left|\gamma_{2 n-1}\right| \leq \frac{1}{2 n}
$$

for all positive integers $n$ with extremal functions of the form (2.8) and (2.9) depending on parity of $n$. 


\section{Logarithmic coefficients for functions in $\mathcal{K}_{s}$}

Theorem 3 If $f \in \mathcal{K}_{S}$, then

$\left|\gamma_{1}\right| \leq \frac{1}{4} \quad, \quad\left|\gamma_{2}\right| \leq \frac{1}{6} \quad, \quad\left|\gamma_{3}\right| \leq \frac{1}{16} \quad, \quad\left|\gamma_{4}\right| \leq \frac{13}{180} \quad, \quad\left|\gamma_{5}\right| \leq \frac{19}{576}+0.0017 \ldots$

The first four bounds are sharp.

Proof Applying the Alexander relation, (2.1) and (1.3) we obtain

$$
\begin{aligned}
& \gamma_{1}=\frac{1}{4} c_{1} \\
& \gamma_{2}=\frac{1}{6}\left(c_{2}+\frac{5}{8} c_{1}^{2}\right) \\
& \gamma_{3}=\frac{1}{16}\left(c_{3}+\frac{5}{3} c_{1} c_{2}+c_{1}^{3}\right) \\
& \gamma_{4}=\frac{1}{20}\left(c_{4}+\frac{11}{8} c_{1} c_{3}+\frac{13}{9} c_{2}^{2}+\frac{205}{72} c_{1}^{2} c_{2}+\frac{251}{288} c_{1}^{4}\right) \\
& \gamma_{5}=\frac{1}{36}\left(c_{5}+\frac{8}{5} c_{1} c_{4}+\frac{9}{4} c_{2} c_{3}+\frac{59}{20} c_{1} c_{2}^{2}+\frac{241}{80} c_{1}^{2} c_{3}+\frac{67}{16} c_{1}^{3} c_{2}+\frac{19}{16} c_{1}^{5}\right)
\end{aligned}
$$

The first two bounds of $\gamma_{1}$ and $\gamma_{2}$ are clear. By Lemma 1 with $\mu=5 / 3$ and $v=1$, there is $\left|\gamma_{3}\right| \leq 1 / 16$.

Now, we write $\gamma_{4}$ as follows

$$
\begin{aligned}
& \gamma_{4}=\frac{1}{5760}[ 198\left(c_{4}+2 c_{1} c_{3}+c_{2}^{2}+3 c_{1}^{2} c_{2}+c_{1}^{4}\right) \\
&\left.+\left(90 c_{4}+218 c_{2}^{2}+226 c_{1}^{2} c_{2}+53 c_{1}^{4}\right)\right] .
\end{aligned}
$$

By Lemma 3, $\left|c_{4}+2 c_{1} c_{3}+c_{2}^{2}+3 c_{1}^{2} c_{2}+c_{1}^{4}\right| \leq 1$. From Lemma 2 and the triangle inequality, we obtain

$$
\begin{aligned}
& \left|90 c_{4}+218 c_{2}^{2}+226 c_{1}^{2} c_{2}+53 c_{1}^{4}\right| \\
& \quad \leq 90\left(1-\left|c_{1}\right|^{2}-\left|c_{2}\right|^{2}\right)+218\left|c_{2}\right|^{2}+226\left|c_{1}\right|^{2}\left|c_{2}\right|+53\left|c_{1}\right|^{4} \\
& \quad=90-90\left|c_{1}\right|^{2}+128\left|c_{2}\right|^{2}+226\left|c_{1}\right|^{2}\left|c_{2}\right|+53\left|c_{1}\right|^{4} \\
& \quad \leq 90-90\left|c_{1}\right|^{2}+128\left(1-\left|c_{1}\right|^{2}\right)^{2}+226\left|c_{1}\right|^{2}\left(1-\left|c_{1}\right|^{2}\right)+53\left|c_{1}\right|^{4} \\
& \quad=218-120\left|c_{1}\right|^{2}-45\left|c_{1}\right|^{4} \leq 218
\end{aligned}
$$

Consequently,

$$
\left|\gamma_{4}\right| \leq \frac{1}{5760}(198+218)=\frac{13}{180} .
$$

The coefficient $\gamma_{5}$ can be rewritten as

$$
\begin{aligned}
\gamma_{5}=\frac{1}{2880}[ & 80\left(c_{5}+2 c_{1} c_{4}+2 c_{2} c_{3}+3 c_{1} c_{2}^{2}+3 c_{1}^{2} c_{3}+4 c_{1}^{3} c_{2}+c_{1}^{5}\right) \\
& \left.+\left(-32 c_{1} c_{4}+20 c_{2} c_{3}+15 c_{1}^{3} c_{2}+c_{1}^{2} c_{3}-4 c_{1} c_{2}^{2}+15 c_{1}^{5}\right)\right] .
\end{aligned}
$$

From Formula (1.7) with $\mu=1$, the first component in the square brackets is 
bounded by 80 . Let us denote the second component by $V$.

Applying the inequalities from Lemma 2 and writing $x=\left|c_{1}\right|, \quad y=\left|c_{2}\right|$, $x, y \in[0,1]$, the expression $V$ can be estimated as follows

$$
\begin{aligned}
|V| & \leq 32 x\left(1-x^{2}-y^{2}\right)+\left(20 y+x^{2}\right)\left(1-x^{2}-\frac{y^{2}}{1+x}\right)+15 x^{3} y+4 x y^{2}+15 x^{5} \\
& \leq 32 x\left(1-x^{2}-y^{2}\right)+\left(20 y+x^{2}\right)\left(1-x^{2}\right)+15 x^{3} y+4 x y^{2}+15 x^{5} .
\end{aligned}
$$

Assume that

$$
G(x, y)=-28 x y^{2}+5\left(4-4 x^{2}+3 x^{3}\right) y+x(32+x)\left(1-x^{2}\right)+15 x^{5}
$$

hence

$$
|V| \leq G(x, y)
$$

In a view of Lemma 2 , the region of variability of a pair $\left(\left|c_{1}\right|,\left|c_{2}\right|\right)$ coincides with a set

$$
\Omega=\left\{(x, y): 0 \leq x \leq 1,0 \leq y \leq 1-x^{2}\right\} .
$$

The critical points of $G$ satisfy the conditions

$$
-28 y^{2}-5 x(8-9 x) y+32+2 x-96 x^{2}-4 x^{3}+75 x^{4}=0 \text { and } y=\frac{5\left(4-4 x^{2}+3 x^{3}\right)}{56 x} .
$$

Solving this system leads to an equality

$$
-400+2784 x^{2}+1424 x^{3}-9552 x^{4}-2848 x^{5}+9525 x^{6}=0,
$$

which has two solutions: $x_{1}=-0.770 \ldots$ and $x_{2}=0.898 \ldots$ Hence, we obtain two critical points: $(-0.770 \ldots,-0.029 \ldots)$ and $(0.898 \ldots, 0.292 \ldots)$. Both points do not belong to $\Omega$.

On the boundary of $\Omega$, we have

$$
\begin{aligned}
G(0, y) & =20 y \leq 20, \\
G(x, 0) & =x\left[32\left(1-x^{2}\right)+x\left(1-x^{2}\right)+15 x^{4}\right]=g_{1}(x), \\
G\left(x, 1-x^{2}\right) & =20+4 x-39 x^{2}+39 x^{3}+19 x^{4}-28 x^{5}=g_{2}(x) .
\end{aligned}
$$

For $g_{1}$, we have

$$
g_{1}^{\prime}(x)=8\left(2-3 x^{2}\right)^{2}+h(x)
$$

with $h(x)=2 x-4 x^{3}+3 x^{4}$. Since $h$ is increasing for $x \in[0,1]$, so $h(x) \geq h(0)=0$. In this way, we have proven that $g_{1}^{\prime}(x) \geq 0$ for $x \in[0,1]$. Consequently,

$$
G(x, 0) \leq g_{1}(1)=15 .
$$

The function $g_{2}$ has in $[0,1]$ the only critical point $x_{0}=0.056 \ldots$, so 


$$
\max \left\{g_{2}(x): x \in[0,1]\right\}=\max \left\{g_{2}(0), g_{2}\left(x_{0}\right), g_{2}(1)\right\}=20.108 \ldots
$$

Summing the bounds in (3.2), we get

$$
\left|\gamma_{5}\right| \leq 0.0347 \ldots
$$

or, in other way,

$$
\left|\gamma_{5}\right| \leq \frac{19}{576}+0.0017 \ldots
$$

Finally, note that the equalities $\left|\gamma_{1}\right|=\frac{1}{4}$ and $\left|\gamma_{3}\right|=\frac{1}{16}$ hold if we take $c_{1}=1$ in (3.2). Similarly, $\left|\gamma_{2}\right|=\frac{1}{6}$ and $\left|\gamma_{4}\right|=\frac{13}{180}$ if we put $c_{2}=1$ into (3.2). This means that the extremal convex functions are

$$
f_{1}(z)=-\log (1-z) \text { and } f_{2}(z)=\frac{1}{2} \log \frac{1+z}{1-z} .
$$

For these functions, the corresponding functions $F$ are following

$$
F_{1}(z)=\frac{1}{4} z+\frac{5}{48} z^{2}+\frac{1}{16} z^{3}+\frac{251}{5760} z^{4}+\frac{19}{576} z^{5}+\ldots
$$

and

$$
F_{2}(z)=\frac{1}{6} z^{2}+\frac{13}{180} z^{4}+\frac{251}{5760} z^{6}+\ldots,
$$

respectively.

For $f_{1}$, we can also see that $\gamma_{5}=\frac{19}{576}$ which is expected sharp bound of $\gamma_{5}$ for all functions in $\mathcal{K}_{S}$.

Author Contributions Not applicable.

Funding The project/research was financed in the framework of the project Lublin University of Technology—Regional Excellence Initiative, funded by the Polish Ministry of Science and Higher Education (contract no. 030/RID/2018/19).

Availability of data and materials Not applicable.

\section{Declarations}

Conflict of interest The authors declare that they have no conflict of interest.

Code availability Not applicable.

Open Access This article is licensed under a Creative Commons Attribution 4.0 International License, which permits use, sharing, adaptation, distribution and reproduction in any medium or format, as long as you give appropriate credit to the original author(s) and the source, provide a link to the Creative Commons licence, and indicate if changes were made. The images or other third party material in this article are included in the article's Creative Commons licence, unless indicated otherwise in a credit line to the material. If material is not included in the article's Creative Commons licence and your intended 
use is not permitted by statutory regulation or exceeds the permitted use, you will need to obtain permission directly from the copyright holder. To view a copy of this licence, visit http:// creativecommons.org/licenses/by/4.0/.

\section{References}

1. Carlson, F.: Sur les coefficients d'une fonction bornée dans le cercle unité, Ark. Mat. Astr. Fys. 27(1), 8, (1940)

2. Efraimidis, I.: A generalization of Livingston's coefficient inequalities for functions with positive real part, J. Math. Anal. Appl. 435(1), 369-379 (2016)

3. Girela, D.: Logarithmic coefficients of univalent functions, Ann. Acad. Sci. Fenn. Math. 25, 337-350 (2000)

4. Obradović, M., Ponnusamy, S., Wirths, K-J.: Logarithmic Coefficients and a Coefficient Conjecture for Univalent Functions, Monatsh. Math. 185(3), 489-501 (2018)

5. Obradović, M., Tuneski, N.: The third logarithmic coefficient for the class $\mathcal{S}$, Turk. J. Math. 44, 1950-1954 (2020)

6. Prokhorov, D.V., Szynal, J.: Inverse coefficients for $(\alpha, \beta)$-convex functions, Ann. Univ. Mariae CurieSkłodowska Sect. A 35, 125-143 (1981)

7. Sakaguchi, K.: On a certain univalent mapping, J. Math. Soc. Jpn. 11, 72-75 (1959)

8. Vasudevarao, A., Thomas, D.K.: The logarithmic coefficients of univalent functions-an overview. In: Current Research in Mathematical and Computer Sciences II, pp. 257-269. Publisher UWM, Olsztyn (2018)

Publisher's Note Springer Nature remains neutral with regard to jurisdictional claims in published maps and institutional affiliations. 